Pacific Journal of Mathematics

SOME INCLUSION THEOREMS FOR SEQUENCE SPACES 


\title{
SOME INCLUSION THEOREMS FOR SEQUENCE SPACES
}

\author{
G. BenNetT
}

\begin{abstract}
The purpose of this paper is to study inclusion theorems for some of the more familiar sequence spaces. Necessary and sufficient conditions are given for an $F K$-space to contain each of the spaces $b v_{0}, b v, \ell^{p}, 0<p<\infty$, and $c_{0}$. It is also shown that the Hardy space $H^{p}, 0<p<1$, is a barrelled subspace of its containing Banach space $B^{p}$. This leads to new results concerning multipliers of $H^{p}$ and to new estimates on the growth of the Taylor coefficients of $B^{p}$ functions.
\end{abstract}

The main results of $\S 2$ give necessary and sufficient conditions for an $F K$-space to contain $\ell, b v_{0}$ or $b v$. These results were obtained by W. H. Ruckle, A. K. Snyder, and A. Wilansky, and the author in a series of seminars held at Lehigh University during the period 19681970. They are rather elementary and probably well-known to other workers in the field; the main reason for including them here (without proof) is that special cases (for example: [7], Theorem 10; [14], Theorems XI (a), (b) and XII (a), (b); [16], Theorem 1 (1); [20], Theorem 1) have been repeatedly established by various authors. Theorems of Dawson [8] and Lorentz and Zeller [19] are extended, and it is shown that $\ell^{p}$ is never a closed subspace of any convergence domain. The results of this section also have relevance to Sember's work on conull $F K$-spaces [22].

$F K$-spaces containing $\ell^{p}, 1<p<\infty$, or $c_{0}$ are characterized in $\S 3$. The techniques employed provide an extension to a result of Singer [23] (for Banach spaces with no subspace isomorphic to $c_{0}$ ) to arbitrary sequentially complete locally convex spaces.

Section 4 covers the spaces $\ell^{p}, 0<p \leqq 1$. It turns out, rather surprisingly, that if an $F K$-space contains one of these spaces, then it contains all of them. As a corollary, it is shown that $\bigcap_{p>0} \ell^{p}$ is a barrelled subspace of $\ell$, thus improving a result of Wilansky ([27], p. 45).

Section 5 returns to the study (see [3]) of scarce copies of solid sequence spaces. $\measuredangle$ and $\omega$ enjoy the rather special property of having every scarce copy barrelled, which fact in turn leads to further inclusion theorems. Similar results are obtained for arbitrary solid $F K$ - $A K$-spaces.

In $\S 6$ a study is made of the Hardy spaces $H^{p}, 0<p<1$, which can be viewed as sequence spaces by identifying functions with their Taylor series. Using a technique of Wilansky ([27], p. 45), it is shown that $H^{p}$ is a barrelled subspace of its containing Banach space $B^{p}$. 
This observation leads to the solution of a problem raised implicitly by Duren, Romberg and Shields in the papers [10], [11] and [12], viz: why do $H^{p}$ and $B^{p}$ almost always have the same multipliers? The paper closes with some new results concerning the size of the Taylor coefficients of functions in $B^{p}$.

1. Notation and preliminary ideas. $\omega$ denotes the space of all (real- or) complex-valued sequences, and any vector subspace of $\omega$ is called a sequence space. A sequence space $E$ with a vector space topology $\tau$ is a $K$-space if the inclusion mapping $(E, \tau) \rightarrow \omega$ is continuous when $\omega$ is endowed with the topology of coordinaterwise convergence. If, in addition, $(E, \tau)$ is complete and metrizable, $(E, \tau)$ is called a Frechet $K$-space; if $\tau$ is locally convex, we abbreviate this to $F K$-space. The basic properties of $F K$-spaces may be found, for example, in [28]. In particular (extending Zeller's theorem 4.5(a) slightly), it follows from the closed graph theorem that if $E$ and $F$ are Frechet $K$-spaces with $E \subseteq F$, then $E$ is continuously embedded in $F$. An $F K$-space whose topology is normable is a $B K$-space.

The following spaces will be important in the sequel: $m$, the space of all bounded sequences; $c$, the space of all convergent sequences; $c_{0}$, the space of null sequences; $\ell^{p}, 0<p<\infty$, the space of all absolutely $p$-summable sequences;

$$
b v=\left\{x \in \omega: \sum_{j=1}^{\infty}\left|x_{j}-x_{j+1}\right|<\infty\right\},
$$

the space of all sequences of bounded variation, which is a $B K$-space under the norm

$$
\|x\|_{b v}=\sum_{j=1}^{\infty}\left|x_{j}-x_{j+1}\right|+\lim _{j \rightarrow \infty}\left|x_{j}\right| \quad(x \in b v) .
$$

As usual, $\ell^{1}$ is replaced by $\ell$, and $b v_{0}$ denotes $b v \cap c_{0}$.

The sequence $(1,1, \cdots)$ is denoted by $e$ and it is clear that $b v=$ $b v_{0}+\{e\} . e^{j}, j=1,2, \cdots$, denotes the sequence $(0, \cdots, 0,1,0, \cdots)$ with the 'one' in the $j$ th position, and $\varphi$ denotes the linear span of $\left\{e^{j}: j=\right.$ $1,2, \cdots\}$ in $\omega$.

We shall also be concerned with matrix transformations $y=A x$, where $x, y \in \omega, A=\left\{a_{i j}\right\}_{i, j=1}^{\infty}$ is an infinite matrix with complex coefficients, and (formally)

$$
y_{i}=\sum_{j=1}^{\infty} a_{i j} x_{j} \quad(i=1,2, \cdots) .
$$

If each of these series converges we say that $y=A x$ exists and write $y \in \omega_{A}$. More generally, if $E$ is any subset of $\omega$, we denote by $E_{A}$ the set 
$\{x \in \omega: A x$ exists and $A x \in E\}$.

$c_{A}$ is called the convergence domain of the matrix $A$ and $x \in c_{A}$ is said to be $A$-limitable. The sequence $\left\{a_{i j}\right\}_{j=1}^{\infty}$ is called the $i$ th row of $A$ and $\left\{a_{i j}\right\}_{i=1}^{\infty}$ the $j$ th column.

The following result, proved by Zeller in [28], Theorem 4.10(a), will be useful throughout the paper.

THEOREM 1. Let $E$ be an $F K$-space whose topology is given by means of the seminorms $\left\{q_{n}\right\}_{n=1}^{\infty}$ and let $A$ be an infinite matrix. Then $E_{A}$ is an $F K$-space when topologized by

$$
\begin{array}{ll}
x \longrightarrow\left|x_{j}\right| & (j=1,2, \cdots) ; \\
x \longrightarrow \sup _{n}\left|\sum_{j=1}^{n} a_{i j} x_{j}\right| & (i=1,2, \cdots) ; \\
x \longrightarrow q_{n}(A x) & (n=1,2, \cdots) .
\end{array}
$$

Finally, if $E$ and $F$ are vector spaces which form a separated dual pair, we follow the notation of [21] and denote the weak topology on $E$ by $\sigma(E, F)$, the Mackey topology by $\tau(E, F)$, and the strong topology by $\beta(E, F)$. If $(E, \tau)$ is a topological vector space, the set of all $\tau$-continuous linear functionals on $E$ is denoted by $E^{\prime}$.

2. $F K$-spaces containing $\ell, b v_{0}$ or $b v$.

Theorem 2. An FK-space $E$ contains $\measuredangle$ if and only if $\left\{e^{j}: j=\right.$ $1,2, \cdots\}$ is a bounded subset of $E$.

Our next result follows from Theorems 1 and 2 by using the fact that $E$ is a $K$-space.

Corollary. Let $A$ be a matrix and $E$ an $F K$-space. Then $A$ maps $\ell$ into $E$ if and only if the columns of $A$ belong to $E$ and form a bounded subset there.

Putting $E=c$ or $\ell^{p}, 1 \leqq p \leqq \infty$, enables us to give 'quickie' proofs of results of Hahn ([14], Theorems XII (a) and (b)), Cohen and Dunford ([7], Theorem 10), and Knopp and Lorentz ([16], Theorem $1(1))$.

Using the result of Hahn, Lorentz and Zeller ([19], Lemma 5) have shown that $l$ cannot be the convergence domain of any matrix $A$. Motivated by their paper, we introduce the set $c_{0}(r, s)=\left\{x \in c_{0}\right.$ : $x_{r_{j}}=-x_{s_{j}}, j=1,2, \cdots ; x_{k}=0$ for other $\left.k\right\}$, where $r=\left\{r_{j}\right\}_{j=1}^{\infty}$ and $s=\left\{s_{j}\right\}_{j=1}^{\infty}$ are sequences of positive integers satisfying $r_{1}<s_{1}<r_{2}<$ $s_{2}<\cdots . c_{0}(r, s)$ is a $B K$-space under the topology generated by the 
norm

$$
x \longrightarrow \sup _{n}\left|x_{r_{j}}\right|
$$

and since $\sum_{k=1}^{s_{j}} x_{k} e^{k} \rightarrow x$ as $j \rightarrow \infty$, it is clear that $\varphi \cap c_{0}(r, s)$ is dense in $c_{0}(r, s)$.

Our next theorem improves the result of Lorentz and Zeller mentioned above and also the results of Dawson [8].

THEOREM 3. If $\left\{A^{n}\right\}_{n=1}^{\infty}$ is a family of matrices, each mapping $\measuredangle$ into $c$, then there exist sequences $r$ and $s$ of positive integers such that

$$
c_{0}(r, s) \subseteq \bigcap_{n=1}^{\infty} c_{A^{n}}
$$

Proof. This is essentially the same as that of Lemma 5 of [19] and will be omitted.

Our next result, which follows immediately from Theorem 3, should be compared with Theorem 4 of [2].

COROLlary 1. If $\left\{A^{n}\right\}_{n=1}^{\infty}$ is a family of matrices, each mapping $\ell$ into $c$, then $\bigcap_{n=1}^{\infty} c_{A^{n}} \backslash \mathbf{U}_{0<p<\infty} \ell^{p}$ is nonempty. In particular, for $0<p<\infty, \ell^{p}$ is not the convergence domain of any matrix.

CoRollary 2. For $1 \leqq p<\infty, \ell^{p}$ is not a closed subspace of any convergence domain.

Proof. Let $p \geqq 1$ be fixed, and suppose that, for some matrix $A$, we have $\ell^{p} \leqq c_{A}$. From Theorem 3 and the remarks made immediately before it, there exist sequences $r$ and $s$ of positive integers such that $c_{0}(r, s) \leqq \bar{\phi}$, the closure of $\varphi$ in $c_{A}$. It follows that $\bar{\varphi} \mid \ell^{p}$ is nonempty, so that $l^{p}$ is not closed in $c_{A}$.

We note that the statement of Corollary 2 is valid whenever $0<p \leqq \infty$. For $0<p<1$ this follows since $\ell^{p}$ is not even locally convex (see also Theorem 6); for $p=\infty, \ell^{\infty}=m$, a separability argument may be used (see [1], Corollary 1 to Theorems 4 and 5).

THEOREM 4. An FK-space contains $b v_{0}(b v)$ if and only if $(e \in E)$ and $\left\{\sum_{j=1}^{n} e^{j}: j=1,2, \cdots\right\}$ is a bounded subset of $E$.

Corollary 1. Let $A$ be a matrix and $E$ and FK-space. Then $A$ maps $b v_{0}$ into $E$ if and only if the columns of $A$ belong to $E$ and their partial sums form a bounded subset there.

Putting $E=c$ or $b v$, enables us to give 'quickie' proofs of results 
of Hahn ([14], Theorems XI (a) and (b)) and Mears ([20], Theorem 1). The important class of conull $F K$-spaces was introduced by Snyder in [24]; an $F K$-space $E$ containing $\varphi \cup\{e\}$ is said to be conull if

$$
\sum_{j=1}^{\infty} e^{j}=e \text { weakly in } E .
$$

The next result, which follows at once from Theorem 4, enables us to remove the hypothesis 'containing $b v$ ' from several of the theorems in [22]. (See also p. 159 of [22].)

Corollary 2. Any conull FK-space must contain bv.

3. $F K$-spaces containing $\ell^{p} 1<p<\infty$, or $c_{0}$. We begin this section in a more general setting by considering a series, $\sum_{i=1}^{\infty} x_{i}$, of elements from a locally convex space $(E, \tau)$. Motivated by [23], p. 131, we say that $\sum_{i=1}^{\infty} x_{i}$ is weakly p-unconditionally Cauchy, $1<$ $p \leqq \infty$, if $\sum_{i=1}^{\infty} y_{i} x_{i}$ converges in $(E, \tau)$ whenever $y \in \ell^{p}$. (Here we use the convention that $\ell^{\infty}=c_{0}$ ) Our next theorem improves a result of Singer ([23], Theorem 8, where the same statement, with $1<p<$ $\infty$, is given, but only for $E$ a Banach space containing no subspace isomorphic to $c_{0}$ ).

Theorem 5. Let $(E, \tau)$ be a sequentially complete locally convex space. Then a series, $\sum_{i=1}^{\infty} x_{i}$, of elements of $E$ is weakly $p$-unconditionally Cauchy, $1<p \leqq \infty$, if and only if

$$
\sum_{i=1}^{\infty}\left|f\left(x_{i}\right)\right|^{q}<\infty \quad\left(f \in E^{\prime}\right)
$$

where $q=p /(p-1)$ denotes the dual exponent of $p$.

Proof. The proof of the necessity of (1) is the same as that given by Singer and will be omitted.

To establish the converse implication, we consider the linear mapping $T$ from $\varphi$ into $E$ defined by

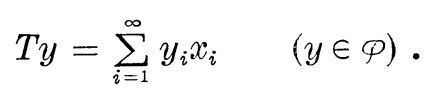

If $F \in E^{\prime}$, then

$$
\langle T y, F\rangle=F\left(\sum_{i=1}^{\infty} y_{i} x_{i}\right)=\sum_{i=1}^{\infty} y_{i} F\left(x_{i}\right)
$$

and so, putting $F\left(x_{i}\right)=f_{i}$, we have $f=\left\{f_{i}\right\}_{i=1}^{\infty} \in \ell^{q}$ and

$$
\langle T y, F\rangle=\langle y, f\rangle \text {. }
$$


It follows that $T$ is $\sigma\left(\varphi, \ell^{q}\right) \rightarrow \sigma\left(E, E^{\prime}\right)$-continuous and so ([21], p. 62) $T$ is also $\tau\left(\varphi, \ell^{q}\right) \rightarrow \tau$-continuous. Now $\ell^{p}$ is $\tau\left(\ell^{p}, \ell^{q}\right)$-metrizable so that $([21]$, p. 82)

$$
\tau\left(\varphi, \ell^{q}\right)=\tau\left(\ell^{p}, \ell^{q}\right) \mid \varphi .
$$

Thus, if $y \in \ell^{p}$, then $\left\{\sum_{j=1}^{n} y_{j} e^{j}\right\}_{n=1}^{\infty}$ is $\tau\left(\varphi, \ell^{q}\right)$-Cauchy so that $\left\{T\left(\sum_{j=1}^{n} y_{j} e^{j}\right)\right\}_{n=1}^{\infty}$ is $\tau$-Cauchy in $E$, i.e., $\left\{\sum_{i=1}^{n} y_{i} x_{i}\right\}_{n=1}^{\infty}$ converges in $E$.

We note that, for $p=\infty$ and $E$ a Banach space, the above result (with different proof) has been given by Bessaga and Pelczynski ([6], Lemma 2).

If $E$ is an $F K$-space containing $\varphi$, then putting $x_{i}=e^{i}, i=1,2$, $\cdots$, gives the following corollary (see also [4], Proposition 5, for the case $p=\infty$ ).

Corollary. An FK-space $E$ contains $\ell^{p}, 1<p \leqq \infty$, if and only if $\varphi \subseteq E$ and

$$
\sum_{j=1}^{\infty}\left|f\left(e^{j}\right)\right|^{q}<\infty \quad\left(f \in E^{\prime}\right),
$$

where $q=p /(p-1)$.

4. $F K$-spaces containing $\ell^{p}, 0<p \leqq 1$. For $0<p<1$ it is wellknown that $\ell^{p}$ is a Frechet $K$-space when topologized by means of the paranorm

$$
x \longrightarrow \sum_{j=1}^{\infty}\left|x_{j}\right|^{p} \quad\left(x \in \ell^{p}\right),
$$

so that ([17], $\$ 15.12(1)) \ell^{p}$ is of the first category in $\ell_{0}$ However, in spite of this, we have the rather surprising

Theorem 6. An FK-space contains $\bigcap_{p>0} \ell^{p}$ (if and) only if it contains $\ell$.

Proof. $\bigcap_{p>0} \ell^{p}$, regarded as a countable intersection, $\bigcap_{n=1}^{\infty} \ell^{1 / n}$, is a Frechet $K$-space when endowed with the projective topology, and $\left\{e^{j}: j=1,2, \cdots\right\}$, being bounded in each $\ell^{p}$ space, is bounded in $\bigcap_{p>0} \ell^{p}$. If $E$ is an $F K$-space containing $\bigcap_{p>0} \ell^{p}$, the inclusion mapping: $\bigcap_{p>0} \ell^{p} \rightarrow E$ is continuous by the closed graph theorem; consequently $\left\{e^{j}: j=1,2, \cdots\right\}$ is bounded in $E$, and the desired conclusion follows from Theorem 2.

COROLlary. $\bigcap_{p>0} \ell^{p}$ is a barrelled subspace of $\iota$.

Proof. Noting that $\bigcap_{p>0} \ell^{p}$ is dense in $\ell$, the corollary follows 
at once from Theorem 1 of [5]. This theorem, however, is rather lengthy, and the following alternative proof of the corollary is perhaps preferable here.

Suppose that $\left\{a^{n}\right\}_{n=1}^{\infty}$ is a $\sigma\left(m, \bigcap_{p>0} \ell^{p}\right)$-bounded sequence of elements of $m$. If $A$ denotes the matrix whose $n$th row is $a^{n}$, then clearly we have $\bigcap_{p>0} \ell^{p} \subseteq m_{A}$. It follows from Theorems 1 and 6 that $\ell \subseteq m_{A}$ so that $\left\{a^{n}\right\}_{n=1}^{\infty}$ is also $\sigma(m, \ell)$-bounded. Thus, the topologies $\sigma\left(m, \bigcap_{p>0} l^{p}\right)$ and $\sigma(m, l)$ define the same bounded sequences and therefore the same bounded sets; consequently,

$$
\beta\left(\bigcap_{p>0} \ell^{p}, m\right)=\beta(\ell, m) \mid \bigcap_{p>0} \ell^{p}=\|\cdot\|_{1} \text {-topology } \mid \bigcap_{p>0} \ell^{p},
$$

and the desired result follows at once from Corollary 1, p. 66, of [21].

The corollary implies that if $X$ is any subspace of $\ell$ with $\bigcap_{p>0} \ell^{p} \subseteq$ $X \subseteq \ell$, then $X$ is a barrelled subspace of $\iota_{0}$ In particular, we obtain the result ([27], Example 1.2, p.45) of Wilansky which asserts that $\ell^{1 / 2}$ is a barelled subspace of $\ell$.

The reader should compare the results of this section with Theorem 8 and its corollary.

5. Scarce copies of sequence spaces. In this section we return to our study of scarce copies of sequence spaces (see [3]) and begin by recalling some definitions. A sequence space $E$ is said to be solid (respectively monotone) if $x y \in E$ whenever $x \in E$ and $y \in m$ (respectively $y_{j}= \pm 1$ for all $j$ ). $r=\left\{r_{n}\right\}_{n=1}^{\infty}$ will always denote a nondecreasing, unbounded sequence of positive integers with $r_{1}=1$ and $r_{n}=o(n)$. We define, for each $x \in \omega$ and each positive integer $n$, the counting function, $c_{n}(x)$, as the number of nonzero elements in the set $\left\{x_{1}, x_{2}\right.$, $\left.\cdots, x_{n}\right\}$. Given any subset $E$ of $\omega$, we consider

$$
\sigma(E, r)=\left\{x \in E: c_{n}(x) \leqq r_{n} \text { for } n=1,2, \cdots\right\} \text {. }
$$

$\sigma(E, r)$ is not, in general, a vector space, and we denote its linear span by $\Sigma(E, r)$. Such a space will be called a scarce copy of $E$; it is clear that if $E$ is solid, monotone, or contains $\varphi$, then every scarce copy of $E$ has the same property. Moreover, if $(E, \tau)$ is a $K$-space containing an element $x$ with $x_{j} \neq 0, j=1,2, \cdots$, then every scarce copy of $E$ is of the first category in $E$.

In view of this last observation the following is somewhat unexpected.

Theorem 7. Every scarce copy of $\omega$ is barrelled.

Proof. Let $\Sigma=\Sigma(\omega, r)$ be a scarce copy of $\omega$. If $K$ is a $\sigma(\varphi, \Sigma)$ bounded subset of $\varphi$, then 


$$
\sup _{a \in K}\left|a_{j}\right|<\infty \quad(j=1,2, \cdots)
$$

since $\Sigma \supseteqq \varphi$. Moreover, it is not difficult to see that there exists a positive integer $j_{0}$ such that

$$
\sup _{a \in K}\left|a_{j}\right|=0 \quad\left(j \geqq j_{0}\right) .
$$

Thus the topologies $\sigma(\varphi, \Sigma)$ and $\sigma(\varphi, \omega)$ define the same bounded sets and the desired result follows as in the proof of the corollary to Theorem 6 .

Theorem 7 may be interpreted as an inclusion result, viz:

Corollary. If an FK-space $E$ contains a scarce copy of $\omega$, then $E=\omega$.

Proof. Noting that every scarce copy of $\omega$ is dense in $\omega$, we may apply Theorem 1, (i) $\Rightarrow$ (ii), of [5] (i.e., the closed graph theorem for barrelled spaces).

In a similar fashion it is possible to establish the following results and we leave the details to the reader.

THEOREM 8. Every scarce copy of $\bigcap_{p>0} \ell^{p}$ is barrelled as a subspace of $\iota$. In particular, every scarce copy of $\ell$ is barrelled.

CoRollary. An FK-space contains a scarce copy of $\bigcap_{p>0} \ell^{p}$ if and only if it contains $\ell$.

Unfortunately, none of the other sequence spaces discussed in this paper $\left(\ell^{p}, 1<p<\infty, b v_{0}, b v, c_{0}, c, m\right)$ possesses this property enjoyed by $\omega$ and $\iota$. Indeed, it can readily be shown that if $X$ is one of these spaces, the no scarce copy of $X$ is barrelled. This observation, along with Theorem 1, (i) $\Leftrightarrow$ (iii), of [5], leads to the following existence result.

Theorem 9. If $X$ is one of the spaces $\ell^{p}, 1<p<\infty, b v_{0}, b v$, $c_{0}, c$ or $m$, and $\Sigma(X, r)$ is a scarce copy of $X$, then there exists a BKspace $Y$ with

$$
\Sigma(X, r) \varsubsetneqq Y \varsubsetneqq X .
$$

However, we do have the following result for $A K$-spaces. (Recall that a topological sequence space $(E, \tau)$ containing $\varphi$ is said to be an $A K$-space provided that $\left\{\sum_{j=1}^{n} x_{j} e^{j}\right\}_{j=1}^{n}$ is $\tau$-convergent to $x$, for each $x \in E)$. 
THeOREM 10. Let $E$ be a solid $F K$-AK-space and let $\Sigma(E)$ denote the union of all the scarce copies of $E$. Then $\Sigma(E)$ is a barrelled subspace of $E$.

Proof. It is an easy consequence of the Banach-Steinhaus theorem that the dual of $E$ may be identified with the space $E^{\alpha}$, where

$$
E^{\alpha}=\left\{y \in \omega: \sum_{j=1}^{\infty}\left|x_{j} y_{j}\right|<\infty, \text { for each } x \in E\right\} .
$$

It follows that the $F K$-topology on $E$ is just $\tau\left(E, E^{\alpha}\right)$, so that ( $E$, $\left.\tau\left(E, E^{\alpha}\right)\right)$ is barrelled. Furthermore, $\Sigma(E)$ may be identified with the sequences $\left\{\lambda_{j} x_{j}\right\}_{j=1}^{\infty}$ where $x \in E$ and $\lambda$ satisfies $\lambda_{j}=0$ or $1, j=1,2, \cdots$, and $\lim _{n \rightarrow \infty} 1 / n \sum_{j=1}^{n} \lambda_{j}=0$. Thus, from a result of Webb ([26], Lemma $\mathrm{D}(1)$, p. 360$), \Sigma(E)$ is $\tau\left(\Sigma(E), E^{\alpha}\right)$-barrelled. (Note that Webb's result was stated only for perfect sequence spaces-the extension to the solid case is routine.) Now, just as in the proof of Theorem 5, we see that $\tau\left(\Sigma(E), E^{\alpha}\right)=\tau\left(E, E^{\alpha}\right) \mid \Sigma(E)$, and it follows that $\Sigma(E)$ is a barrelled subspace of $E$.

Corollary. Let $E$ be a given solid $F K$-AK-space. Then an $F K$ space contains every scarce copy of $E$ if and only if it contains $E$.

6. $H^{p}, 0<p<1$, and its containing Banach space. In this section we consider the Hardy space $H^{p}, 0<p<1$, of functions $f$ which are analytic in the open unit disk and which satisfy

$$
\|f\|_{H^{p}}=\sup _{0<r<1} \frac{1}{2 \pi} \int_{0}^{2 \pi}\left|f\left(r e^{i \theta}\right)\right|^{p} d \theta<\infty .
$$

It is a well-known result of Walters ([25], Theorem) that $H^{p}$ is a Frechet space under the paranorm $\|\cdot\|_{p}$. We shall also be interested in the space $B^{p}, 0<p<1$, of functions analytic in the disk which satisfy

$$
\|f\|_{B^{p}}=\frac{1}{2 \pi} \int_{0}^{1}(1-r)^{1 / p-2}\left(\int_{0}^{2 \pi}\left|f\left(r e^{i \theta}\right)\right| d \theta\right) d r<\infty
$$

For $0<p<1$, it is known that $H^{p} \subset B^{p}$ ([15], p. 412), that $B^{p}$ is a Banach space under the norm $\|\cdot\|_{B^{p}}\left([10]\right.$, Theorem 3), and that $H^{p}$ is dense in $B^{p}$ ([10], Theorem 3 (iii)).

For a subset $E$ of $\omega, \lambda \in \omega$ is said to be a multiplier of $H^{p}$ (respectively $B^{p}$ ) into $E$ if whenever

$$
f(z)=\sum_{n=0}^{\infty} a_{n} z^{n} \in H^{p} \quad\left(\text { respectively } B^{p}\right),
$$


then $\left\{\lambda_{n} a_{n}\right\}_{n=0}^{\infty} \in E$. The theory of multipliers has been studied extensively by various authors and we refer the reader to [9], §6.4, for the details. The purpose of this section is to show that $H^{p}$ and $B^{p}$ 'nearly always' have the same multipliers, thus answering a question raised implicitly by the work of Duren, Romberg and Shields in the papers [10], [11] and [12].

For the remainder of this section it will be convenient for us to regard $H^{p}$ and $B^{p}$ as sequence spaces. This we do, as usual, by identifying functions analytic in the disk with their Taylor coefficients.

Proposition 1. Under the identification mentioned above, $B^{p}$ is a BK-space and $H^{p}$ a Frechet $K$-space.

Proof. It follows from the remarks made above that $B^{p}$ is a Banach sequence space. To show that the coordinate mappings are continuous, we consider

$$
f(z)=\sum_{n=0}^{\infty} a_{n} z^{n} \in B^{p}
$$

Fixing $n$, we have

$$
\begin{aligned}
& \left|a_{n}\right| \beta\left(n+1, \frac{1}{p}-1\right)=\int_{0}^{1} r^{n}(1-r)^{1 / p-2}\left|a_{n}\right| d r \\
\leqq & \left.\frac{1}{2 \pi} \int_{0}^{1}(1-r)^{1 / p-2} \int_{0}^{2-\pi}\left|f\left(r e^{i \theta}\right)\right| d \theta\right) d r \\
& \text { by the Cauchy integral formula } \\
= & \|f\|_{B^{p}} .
\end{aligned}
$$

Consequently, $B^{p}$ is a $B K$-space.

That $H^{p}$ is a $K$-space is a well-known result ([25], Theorem 6) due to Walters.

We note here that the constant $\beta(n+1,1 / p-1)$ given in the proof of Proposition 1 is best possible. This can be seen by considering the functions $f(z)=1, z, z^{2}$, etc. As a corollary, we obtain the following slight sharpening of a result of Duren, Romberg and Shields and Shields ([10], Theorem 4).

CoRollary. If $f(z)=\sum_{n=0}^{\infty} a_{n} z^{n} \in B^{p}$, then

$$
\left|a_{n}\right| \leqq\left(\frac{1}{p}-1\right)(n+1)^{1 / p-1}\|f\|_{B^{p}}
$$

Proof. Note that 


$$
\begin{aligned}
& \beta\left(n+1, \frac{1}{p}-1\right)=\frac{\Gamma(n+1 / p)}{\Gamma(n+1) \Gamma(1 / p-1)} \\
= & \frac{(1 / p-1) 1 / p(1 / p+1) \cdots(1 / p+n-1)}{n !} \leqq(1 / p-1)(n+1)^{1 / p-1} .
\end{aligned}
$$

Our next result is based on idea of Wilansky ([27], Example 1.2 , p. 45).

LeMma 1. Let $(E, \tau)$ be a metrizable locally convex space and let $F$ be a subspace of $E$. Suppose there exists a Frechet topology $\tau_{1} \geqq$ $\tau \mid F$ on $F$ with the property that every $\tau_{1}$-continuous linear functional is $\tau \mid F$-continuous. Then $(F|\tau| F)$ is barrelled.

Proof. Given a barrel, $V$, in $(F, \tau \mid F)$, we are required to show that $V$ is a $\tau \mid F$-neighborhood of zero. Now $V$, being absorbent, we have $F=\bigcup_{n=1}^{\infty} n V$. Each $n V$, being $\tau \mid F$-closed, must also be $\tau_{1}$-closed, and it follows from the Baire category theorem that one of them must contain a $\tau_{1}$-open set. Thus $V$ itself is a convex $\tau_{1}$-neighborhood of zero.

We complete the proof by showing that every convex $\tau_{1}$-neighborhood of zero is a $\tau \mid F$-neighborhood of zero. To do this, we consider the locally convex topology $\tau_{2}$ generated by $\tau_{1}$. (A base for this topology is formed by taking convex covers of $\tau_{1}$-neighborhoods of zero.) Clearly, we have

$$
\tau \mid F \leqq \tau_{2} \leqq \tau_{1}
$$

and so our hypothesis imply that $\left(F, \tau_{2}\right)^{\prime}=(F, \tau \mid F)^{\prime}$. But $\tau$ is metrizable so that ([21], p. 82) $\tau \mid F$ is just the Mackey topology. Consequently

$$
\tau_{2} \leqq \tau \mid F,
$$

and the inclusion relationships (2) and (3) give the desired result.

Theorem 11. $H^{p}$ is a barrelled subspace of $B^{p}, 0<p<1$.

Proof. We take $E=B^{p}$ and $F=H^{p}$ in the lemma. That $\tau_{1} \geqq$ $\tau \mid F$ follows from Proposition 1 and the closed graph theorem for complete metric spaces (see, for example, [17], $\$ 15.12(3)$ ). That $\tau_{1}$ and $\tau \mid F$ define the same continuous linear functionals is just Theorem 7 of [10].

We note that the proof of the lemma also gives the following information-the locally convex topology on $H^{p}$ generated by $\|\cdot\|_{H^{p}}$ is just the subspace topology from $B^{p}$. This observation leads to a slight improvement of a result ([18], p. 614) of Livingston-the convex cover of $a\|\cdot\|_{H^{p}}$-ball contains an entire $\|\cdot\|_{B^{p}}$-ball. 
We are now in a position to state the main result of this section.

Theorem 12. An FK-space contains $H^{p}$ if and only if contains $B^{p}$.

Proof. The sufficiency being obvious, we restrict attention to the necessity. But this follows as in the proof of the corollary to Theorem 7 , since, by Theorem 11 and the remarks made at the beginning of this section, $H^{p}$ is a dense barrelled subspace of $B^{p}$.

Corollary 1. Let $A$ be a matrix and $E$ an $F K$-space. Then $A$ maps $H^{p}$ into $E$ if and only if $A$ maps $B^{p}$ into $E$.

Proof. $A$ maps $H^{p}$ into $E \Longleftarrow H^{p} \subseteq E_{A}$

$\Longrightarrow B^{p} \subseteq E_{A}$ (by Theorems 1 and 12)

$\Longleftrightarrow A$ maps $B^{p}$ into $E$.

Our next result answers a question raised implicitly by the work of Duren, Romberg and Shields in [10], [11] and [12].

COROLlary 2. $H^{p}$ and $B^{p}$ have the same multipliers into any FK-space.

Proof. This follows at once from Corollary 1 noting that coordinaterwise multiplication by $\lambda$ is given by means of the matrix $A$, where $a_{i j}=\delta_{i j} \lambda_{j}$.

Finally, we indicate how Theorem 12 may be used to give new estimates for the Taylor coefficients of $B^{p}$ functions. If $0<p<1$ and

$$
f(z)=\sum_{n=0}^{\infty} a_{n} z^{n} \in H^{p},
$$

well-known results of Hardy and Littlewood ([9], Theorems 6.2 and 6.4) assert that

$$
\sum_{n=1}^{\infty} n^{p-2}\left|a_{n}\right|^{p}<\infty
$$

and

$$
a_{n}=0\left(n^{1 / p-1}\right) .
$$

We note here that (5) does not follow from (4), contrary to the assertion made on page 421 of [13]. Duren, Romberg and Shields ([11], p. 259) point out that (4) may fail for $B^{p}$ functions, but ([10], Theorem 4) that (5) carries over to this larger class. (The latter observation follows at once from Theorem 12.) Combining (4) and (5) give a whole family of mutually independent inequalities, namely 


$$
\sum_{n=1}^{\infty} n^{(p q-p-q) / p}\left|a_{n}\right|^{q}<\infty
$$

whenever $q>p$.

Duren and Shields ([11], p. 259) have observed that (6) carries over to $B^{p}$ functions if $q=1$, but fails whenever $q<1$. We complete the picture by establishing

CoRollary 3. If $f(z)=\sum_{n=0}^{\infty} a_{n} z^{n} \in B^{p}$, then

$$
\sum_{n=1}^{\infty} n^{(p q-p-q) / p}\left|a_{n}\right|^{q}<\infty
$$

whenever $q \geqq 1$.

Proof. Letting $E=\left\{x \in \omega: \sum_{n=1}^{\infty} n^{(p q-p-q) / p}\left|a_{n}\right|^{q}<\infty\right\}$, it is clear that $E$ is a $B K$-space whenever $q \geqq 1$. Inequality (7) asserts that $H^{p} \cong E$; Theorem 12 gives $B^{p} \subseteq E$, and inequality (7) is established.

Added in proof. In a private communication, Professor P. L. Duren has kindly pointed out that Corollary 3 also follows from the special case $q=1$ and the inequality (5).

\section{REFERENCES}

1. G. Bennett, A representation theorem for summability domains, Proc. London Math. Soc., (3) 24 (1972), 193-203.

2. ㄴ The gliding humps technique for FK-spaces, Trans. Amer. Math. Soc., 166 (1972), 285-292.

3. - A new class of sequence spaces with applications in summability theory,

J. Reine Angew. Math., to appear.

4. G. Bennett and N. J. Kalton, FK-spaces containing $c_{0}$, Duke Math. J., 39 (1972), $561-582$.

5. — Inclusion theorems for K-spaces, Canad. J. Math., to appear.

6. C. Bessaga and A. Pelczynski, On bases and unconditional convergence of series in Banach spaces, Studia Math., 17 (1958), 151-164.

7. L. W. Cohen and N. Dunford, Transformations on sequence spaces, Duke Math. J., 3 (1937), 689-701.

8. D. F. Dawson, Linear methods which sum sequences of bounded variation, Proc. Amer. Math. Soc., 17 (1966), 345-348.

9. P. L. Duren, Theory of $H^{p}$ Spaces, Academic Press, New York, 1970.

10. P. L. Duren, B. W. Romberg and A. L. Shields, Linear functionals on $H^{p}$ spaces with $0<p<1$, J. Reine Angew. Math., 238 (1969), 32-60.

11. P. L. Duren and A. L. Shields, Properties of $H^{p}(0<p<1)$ and its containing Banach space, Trans. Amer. Math. Soc., 141 (1969), 255-262.

12. - Coefficient multipliers of $H^{p}$ and $B^{p}$ spaces, Pacific J. Math., 32 (1970), 69-78.

13. P. L. Duren and G. D. Taylor, Mean growth and coefficients of $H^{p}$ functions, Illinois J. Math., 14 (1970), 419-423.

14. H. Hahn, Über Folgen linearer Operationen, Monatsh. für Math., 32 (1922), 3-88. 
15. G. H. Hardy and J. E. Littlewood, Some properties of fractional integrals, II, Math. Z., 34 (1932), 403-439.

16. K. Knopp and G. G. Lorentz, Beiträge zur absoluten Limitierung, Arch. Math., 2 (1949), 10-18.

17. G. Köthe, Topological Vector Spaces, I, Springer, New York, 1969.

18. A. E. Livingston, The space $H^{p}, 0<p<1$, is not normable, Pacific J. Math., 3 (1953), 613-616.

19. G. G. Lorentz and K. Zeller, Strong and ordinary summability, Tohoku Math. J., 15 (1963), 315-321.

20. F. M. Mears, Absolute regularity and the Norlund mean, Ann. Math., 38 (1937), 594-601.

21. A. P. Robertson and W. J. Robertson, Topological Vector Spaces, Cambridge University Press, Cambridge, 1964.

22. J. J. Sember, Variational FK-spaces and two-norm convergence, Math. Z., 119 (1971), 153-159.

23. I. Singer, Some remarks on domination of sequences, Math. Ann., 184 (1970), 113-132.

24. A. K. Snyder, Conull and coregular FK-spaces, Math. Z., 90 (1965), 376-381.

25. S. S. Walters, The space $H^{p}$ with $0<p<1$, Proc. Amer. Math. Soc., 1 (1950), 800-805.

26. J. H. Webb, Sequential convergence in locally convex spaces, Proc. Camb. Phil. Soc., 64 (1968), 341-364.

27. A. Wilansky, Topics in Functional Analysis, Springer lecture notes, no. 45 Berlin, 1967.

28. K. Zeller. Allgemeine Eigenschaften von Limitierungsverfahren, Math. Z., 53 (1951), 463-487.

Received February 4, 1972.

INDIANA UNIVERSITY 


\section{PACIFIC JOURNAL OF MATHEMATICS}

\section{EDITORS}

D. Gilbarg and J. Milgram Stanford University

Stanford, California 94305

R. A. Beaumont

University of Washington

Seattle, Washington 98105
J. DugundJI

Department of Mathematics

University of Southern California

Los Angeles, California 90007

RICHARD ARENS

University of California

Los Angeles, California 90024

ASSOCIATE EDITORS
E. F. BECKENBACH
B. H. NeumanN
F. WOLF
K. YoSHIDA

\section{SUPPORTING INSTITUTIONS}

UNIVERSITY OF BRITISH COLUMBIA

CALIFORNIA INSTITUTE OF TECHNOLOGY

UNIVERSITY OF CALIFORNIA

MONTANA STATE UNIVERSITY

UNIVERSITY OF NEVADA

NEW MEXICO STATE UNIVERSITY

OREGON STATE UNIVERSITY

UNIVERSITY OF OREGON

OSAKA UNIVERSITY
UNIVERSITY OF SOUTHERN CALIFORNIA

STANFORD UNIVERSITY

UNIVERSITY OF TOKYO

UNIVERSITY OF UTAH

WASHINGTON STATE UNIVERSITY

UNIVERSITY OF WASHINGTON

$\stackrel{*}{*} \stackrel{*}{*} \stackrel{*}{*}$ AMERICAN MATHEMATICAL SOCIETY

NAVAL WEAPONS CENTER 


\section{Pacific Journal of Mathematics}

\section{Vol. 46, No. $1 \quad$ November, 1973}

Allan Francis Abrahamse, Uniform integrability of derivatives on $\sigma$-lattices .......................................... 1

Ronald Alter and K. K. Kubota, The diophantine equation $x^{2}+D=p^{n} \ldots \ldots \quad 11$

Grahame Bennett, Some inclusion theorems for sequence spaces .......... 17

William Cutler, On extending isotopies ........................ 31

Robert Jay Daverman, Factored codimension one cells in Euclidean

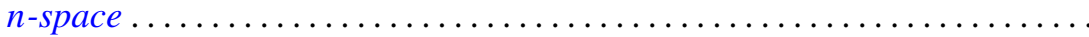

Patrick Barry Eberlein and Barrett O’Neill, Visibility manifolds ............ 45

M. Edelstein, Concerning dentability .......................... 111

Edward Graham Evans, Jr., Krull-Schmidt and cancellation over local

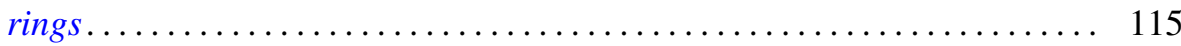

C. D. Feustel, A generalization of Kneser's conjecture ................ 123

Avner Friedman, Uniqueness for the Cauchy problem for degenerate parabolic equations .......................................... 131

David Golber, The cohomological description of a torus action ............ 149

Alain Goullet de Rugy, Un théorème du genre "Andô-Edwards" pour les

Fréchet ordonnés normaux..............................

Louise Hay, The class of recursively enumerable subsets of a recursively enumerable set ........................................

John Paul Helm, Albert Ronald da Silva Meyer and Paul Ruel Young, On orders of translations and enumerations...

Julien O. Hennefeld, A decomposition for $B(X)^{*}$ and unique Hahn-Banach

extensions

Gordon G. Johnson, Moment sequences in Hilbert space .

Thomas Rollin Kramer, A note on countably subparacompact spaces ...

Yves A. Lequain, Differential simplicity and extensions of a derivation ....

Peter Lorimer, A property of the groups Aut $\mathrm{PU}\left(3, q^{2}\right) \ldots$

225

Yasou Matsugu, The Levi problem for a product manifold.

231

John M.F. O'Connell, Real parts of uniform algebras ......

William Lindall Paschke, A factorable Banach algebra without bounded

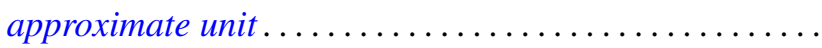

Ronald Joel Rudman, On the fundamental unit of a purely cubic field ....

Tsuan Wu Ting, Torsional rigidities in the elastic-plastic torsion of simply connected cylindrical bars .........................

Philip C. Tonne, Matrix representations for linear transformations on analytic sequences...................................

Jung-Hsien Tsai, On E-compact spaces and generalizations of perfect

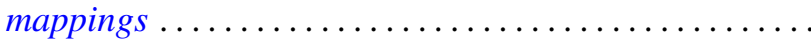

Alfons Van Daele, The upper envelope of invariant functionals majorized by an invariant weight. .. 